\title{
Nutrición y medios de comunicación. ¿Podemos distinguir la información veraz de los mitos? Estudio piloto en población adultos y adolescentes
}

\section{Nutrition and the media. Can we distinguish true information from myths? Pilot study in adults and adolescents population}

\author{
Andrea de Lucas-Muñoza, María Ángeles Martínº, Marta Mesíasa,b \\ a Departamento de Nutrición y Ciencia de los Alimentos, Universidad Complutense de Madrid, España \\ b Instituto de Ciencia y Tecnología de Alimentos y Nutrición (ICTAN-CSIC), España
}

\section{Resumen}

Introducción: La alimentación y la nutrición son ámbitos de gran interés para la población debido a su relación con la salud, la estética y el medioambiente. Este interés va ligado al aumento de información sobre estos temas en los medios de comunicación. Sin embargo, en muchos casos, la información transmitida no es al $100 \%$ veraz, conduciendo a falsas creencias y a la creación de los mitos alimentarios. Objetivos: Realizar un estudio piloto con el fin de evaluar el conocimiento de un grupo de sujetos (adultos y adolescentes) sobre determinados mitos alimentarios y los medios más utilizados para obtener dicho conocimiento. Metodología: Se elaboró una encuesta, distribuida electrónicamente a 200 participantes. Resultados: Casi un $80 \%$ admitió buscar información sobre alimentación de forma voluntaria, utilizando fundamentalmente internet y visitando principalmente redes sociales como Instagram, Facebook o Twitter. Conclusión: A pesar de que la población adulta y adolescente no ha mostrado una gran confusión en relación a determinados mitos alimentarios, es importante concienciar a la sociedad de que hay que tener espíritu crítico y contrastar la información transmitida con el fin de erradicar mitos y noticias falsas sobre aspectos relacionados con la alimentación y la nutrición.

Palabras clave: Alimentación; nutrición; mitos; medios de comunicación; redes sociales.

\begin{abstract}
Introduction: Food and nutrition are areas of great interest to the population, due to their relationship with health, aesthetics and the environment. This interest is linked to the increase in information on these issues in the media. However, in many cases, the information transmitted is not $100 \%$ true, leading to false beliefs and the creation of food myths. Objectives: To carry out a pilot study to evaluate the knowledge of a group of subjects (adults and adolescents) about certain food myths and the most used means to obtain such knowledge. Methodology: A survey was prepared, distributed electronically to 200 participants. Results: Around $80 \%$ admitted to seek information on food voluntarily, mainly using internet and mainly visiting social networks such as Instagram, Facebook or Twitter. Conclusions: Despite the fact that the adult and adolescent population has not shown great confusion in relation to certain food myths, it is important to make society aware that it is necessary to be critical and to contrast the information transmitted in order to eradicate myths and false news about aspects related to food and nutrition.
\end{abstract}

Key words: Food; nutrition; myths; the media; social networks. 


\section{Introducción}

$\mathrm{H}$ oy en día la sociedad es bombardeada constantemente con información sobre alimentación y nutrición a través de diversos medios de comunicación como televisión, radio, y de forma exponencial, internet en sus distintos formatos (redes sociales, blogs, prensa electrónica, páginas especializadas, etc.). Además, en las últimas décadas, se ha observado una clara influencia de los medios de comunicación en los hábitos alimentarios de la población (Carretero-Sánchez, 2016).

El aumento de información sobre alimentación y nutrición ha ido en paralelo con el interés de la sociedad actual en estos ámbitos. Según la Encuesta de Percepción Social de la Ciencia, el porcentaje de personas que muestra interés en el apartado "alimentación y consumo" crece sin cesar. En 2018, el $19 \%$ de los españoles indicó específicamente que "la alimentación y el consumo" es uno de los temas que más atraen su atención (Fundación Española para la Ciencia y la Tecnología, 2018). Además, los españoles han aumentado el interés por la cocina y la alimentación, destacando que un 23\% de la población utiliza aplicaciones para dispositivos móviles sobre estos temas (Barreiro, 2019). El informe Healthy Food \& Brands, publicado en 2019, afirmó que un 74\% de la población española considera importante alimentarse de forma sana. Según este informe, los principales motivos para seguir hábitos alimentarios saludables son proteger la salud, razones médicas o estéticas, el cuidado personal y salvaguardar el medio ambiente (Echenove \& Puértolas, 2019).

Identificar el mecanismo por el cual la sociedad se informa sobre alimentación y nutrición es un proceso complejo, debido a que no se recibe información por un único medio. Accedemos a las noticias no sólo a través de los medios de comunicación, sino que es muy frecuente que en nuestro entorno se comente o se hagan críticas hacia ciertas conductas alimentarias sin realmente tener conocimientos previos. Por todo ello, es importante que los datos transmitidos estén contrastados científicamente. Sin embargo, en muchos casos, esto no se cumple.

La divulgación de información errónea puede dar lugar a falsas creencias y, por consiguiente, a la aparición de diferentes mitos y bulos sobre los alimentos. Un mito alimentario es una creencia popular falsa sobre alimentación que acaba frecuentemente en tendencia 0 incluso en hábito alimentario (Feldman \& Marks, 2016). En muchas ocasiones deriva del desconocimiento o la ignorancia, pero también puede asociarse a creencias erróneas fomentadas por intereses comerciales, económicos y sociales. Las distintas opiniones sobre un mismo tema conducen a confusión y a no saber distinguir la realidad de la fantasía (Castillo Sánchez et al., 2001).

El origen de los mitos puede estar asociado al gran poder de la publicidad engañosa, presentando sus productos como algo milagroso o haciendo creer que tienen ciertas características beneficiosas para la salud cuando realmente no es así. Se espera que para el año 2022, la mitad de las noticias que se emitan en las redes sociales sean falsas, surgiendo la corriente de falsa información denominada fake news. Actualmente el $30 \%$ de las noticias falsas trata sobre alimentación, pudiendo hacer cambiar los hábitos alimentarios al $57 \%$ de los consumidores (Ipsos, 2018). Además, no sólo es importante vigilar cuidadosamente las noticias que se emiten en los medios de comunicación, sino las connotaciones que llevan implícitas, ya que el receptor de la información puede malinterpretarla. Por tanto, es imprescindible que, en noticias con gran impacto social, el contenido sea riguroso y, sobre todo, de calidad (Alzate Narváez, 2013).

El objetivo principal de este estudio piloto fue evaluar el conocimiento de un grupo de sujetos (adultos y adolescentes) sobre ciertos mitos relacionados con la alimentación y la nutrición, así como identificar los medios más utilizados para adquirir esa información.

\section{Métodos}

\section{Diseño y contexto}

Se realizó un estudio piloto de tipo observacional, transversal, descriptivo utilizando la encuesta como técnica de investigación. Se diseñó un cuestionario ad hoc, mediante la herramienta Formularios de Google Forms. La encuesta incluyó 10 preguntas divididas en dos secciones: (1) características sociodemográficas (sexo y edad) y (2) cuestionario sobre mitos alimentarios y posibles fuentes de información, siendo las respuestas cerradas y semicerradas o semiabiertas (Tabla 1). Los mitos incluidos fueron seleccionados a partir de la información descrita por Sánchez (2016) en relación a mitos sobre nutrición. 
Tabla 1. Cuestionario “¿Cuánto sabes de nutrición?, ¿Quién te lo ha contado?”

\begin{tabular}{|c|c|}
\hline Datos sociodemográficos & \\
\hline 1. Sexo & Hombre - Mujer \\
\hline Edad & $\begin{array}{l}15-20 \text { años - 21-30 años } \\
31-40 \text { años - > } 41 \text { años }\end{array}$ \\
\hline \multicolumn{2}{|l|}{ Cuestionario } \\
\hline $\begin{array}{l}\text { 2. ¿Busca información en internet en aspectos } \\
\text { relacionados con la nutrición? }\end{array}$ & $\begin{array}{l}\text { - Sí } \\
\text { - No }\end{array}$ \\
\hline En caso afirmativo, indique dónde busca dicha información: & $\begin{array}{l}\text { Redes sociales (Facebook, Instagram, Twitter....) } \\
\text { Blogs de nutrición } \\
\text { Páginas especializadas } \\
\text { Periódicos digitales } \\
\text { Otros... }\end{array}$ \\
\hline 3. ¿Las dietas sin gluten son más saludables? & $\begin{array}{l}\text { - Sí, porque el gluten daña el tracto digestivo } \\
\text { - Sólo en el caso de las personas intolerantes al gluten } \\
\text { - No, en ningún caso }\end{array}$ \\
\hline $\begin{array}{l}\text { 4. Considera que las grasas ingeridas en la dieta son } \\
\text { perjudiciales? }\end{array}$ & $\begin{array}{l}\text { - Sí, todas las grasas son perjudiciales } \\
\text { - Depende del tipo de grasa y de su cantidad. } \\
\text { - No, sólo la grasa de palma. }\end{array}$ \\
\hline 5. ¿Cree que los alimentos light son más saludables? & $\begin{array}{l}\text { - Sí, porque tienen menos calorías } \\
\text { - Depende del tipo alimento } \\
\text { - No, porque "saludable" no depende de las calorías del alimento sino de } \\
\text { su composición }\end{array}$ \\
\hline 6. El desayuno es la comida más importante del día? & $\begin{array}{l}\text { - Sí, siempre y da igual lo que consumas porque lo quemas durante el día } \\
\text { - Depende de cada persona y de la elección de los alimentos } \\
\text { - No, nunca, porque ya tenemos energía acumulada en el organismo }\end{array}$ \\
\hline 7. ¿Es obligatorio comer 5 veces al día? & $\begin{array}{l}\text { - Sí, sin excepción para que se acelere el metabolismo } \\
\text { - No, depende de la rutina diaria de cada individuo } \\
\text { - No, nunca }\end{array}$ \\
\hline 8. ¿Los huevos aumentan el colesterol? & $\begin{array}{l}\text { - Sí, siempre } \\
\text { - Sólo a partir de más de tres huevos por semana. } \\
\text { - No, depende del resto de la dieta }\end{array}$ \\
\hline $\begin{array}{l}\text { 9. ¿La leche sin lactosa es más saludable que la que sí la } \\
\text { contiene? }\end{array}$ & $\begin{array}{l}\text { - Sí, la lactosa daña el tracto digestivo } \\
\text { - Sólo en el caso de las personas intolerantes a la lactosa } \\
\text { - No, en ningún caso }\end{array}$ \\
\hline 10. Indique dónde ha obtenido esta información: & $\begin{array}{l}\text { Internet: redes sociales, blogs, periódicos digitales, páginas } \\
\text { especializadas. } \\
\text { Tv-radio } \\
\text { Familia-amigos } \\
\text { Formación personal } \\
\text { Profesionales de la salud } \\
\text { Otra.... }\end{array}$ \\
\hline
\end{tabular}


Muestra y participantes

La encuesta se difundió a través de correo electrónico, Whatsapp, Instagram y Facebook, durante el periodo comprendido entre el 14 y el 25 de marzo de 2020. El tipo de muestreo utilizado fue no probabilístico por bola de nieve, donde se selecciona a un primer grupo de individuos y éstos, a su vez, reclutan a nuevos participantes entre sus conocidos y así sucesivamente, hasta conseguir el número de encuestados deseado (Berg, 1988). El primer grupo de individuos estuvo formado por 20 sujetos conocidos de los investigadores, siendo el criterio de selección: (1) la edad (entre 15 y más de 41 años), (2) el sexo (10 hombres y 10 mujeres) y (3) que fueran personas sin un especial conocimiento en el ámbito de la alimentación y la nutrición. El propósito de estos criterios fue abarcar un grupo poblacional heterogéneo, perteneciente a un amplio rango de edad y de distintos géneros. Todo ello para intentar conseguir representatividad y evitar obtener resultados sesgados debido a una especial formación relacionada con la temática de la encuesta. Se estimó el tamaño muestral teniendo en cuenta las especificaciones descritas por García-García et al. (2013) para este tipo de estudios.

\section{Análisis de los datos}

Una vez cerrado el formulario, la información recibida a través de las encuestas se utilizó para agrupar los resultados cualitativos. Los datos se analizaron utilizando el programa Microsoft Excel. El estudio no pretendió obtener significación estadística, sino definir los patrones generales de las respuestas, teniendo en cuenta la heterogeneidad de la muestra para el grupo poblacional encuestado (población adulta y adolescente).

\section{Resultados}

\section{Características de la población encuestada}

Un total de 200 participantes realizaron la encuesta del presente estudio. Hubo una gran diferencia de participación con respecto al sexo, siendo 162 personas (81\%) mujeres y el resto (19\%) hombres. La edad de los encuestados osciló entre 15-20 años (25,5\%), 21-30 años (7,0\%), 31-40 años $(33,0 \%)$ y mayores de 41 años $(34,5 \%)$.

Obtención de información respecto al ámbito de la nutrición

Entre los participantes, 155 (78\%) afirmaron buscar de forma voluntaria información en internet sobre aspectos relacionados con la alimentación y la nutrición, utilizando para ello principalmente las redes sociales (Facebook, Instagram o Twitter, entre otras), seguidas de páginas especializadas, blogs de nutrición y periódicos digitales (Figura 1). No se encontraron diferencias significativas en cuanto al sexo de los sujetos. El uso de canales youtube, programas de cocina y artículos científicos son otras alternativas propuestas por los encuestados como búsqueda de información.

Opinión sobre mitos relacionados con la alimentación y la nutrición

El cuestionario incluyó siete preguntas relacionadas con distintos mitos alimentarios, descritos por Sánchez (2016) y una última pregunta sobre la fuente de obtención de esa información (Tabla 1).

Mito 1. ¿Las dietas sin gluten son más saludables? Un $80,5 \%$ de los encuestados seleccionaron la respuesta: "sólo en el caso de las personas intolerantes al gluten" (Figura 2A). Un 8\% pensaba que el gluten daña el tracto digestivo, mientras que el resto $(11,5 \%)$ afirmó que en ningún caso las dietas sin gluten eran más saludables.

Mito 2. ¿Considera que las grasas ingeridas en la dieta son perjudiciales? Prácticamente todos los participantes $(97,5 \%)$ acertaron con la respuesta sobre este mito (Figura 2A), indicando que "depende del tipo de grasa y de su cantidad". Sólo el 2,5\% seleccionó que todas las grasas de la dieta eran perjudiciales y ninguno de ellos respondió que sólo la grasa de palma es la perjudicial.

Mito 3. ¿Cree que los alimentos light son más saludables? En este mito, el $75 \%$ de los encuestados respondieron negativamente sobre este mito (Figura 2A), seleccionando la opción "no, porque saludable no depende de las calorías del alimento sino de su composición".

Mito 4. ¿El desayuno es la comida más importante del día? La respuesta ("depende de cada persona y de la elección de los alimentos") fue seleccionada por el 64,5\% de los sujetos encuestados (Figura 2A). Es importante destacar que el 34,5\% eligió la respuesta: "sí, siempre, y da igual lo que consumas porque lo quemas durante el día", confirmando la creencia de este mito entre la población. Sólo un 1\% optó por la opción "no, nunca, porque ya tenemos energía acumulada en el organismo". Mito 5. ¿Es obligatorio comer 5 veces al día? Un 63\% de los participantes afirmó que "No, depende de la rutina de cada individuo" (Figura $2 \mathrm{~A}$ ). De nuevo, en esta ocasión, el mito está presente entre la población, ya que el $36,5 \%$ respondió que "sí, sin excepción" y sólo un 0,5\% seleccionó que "no, nunca". 


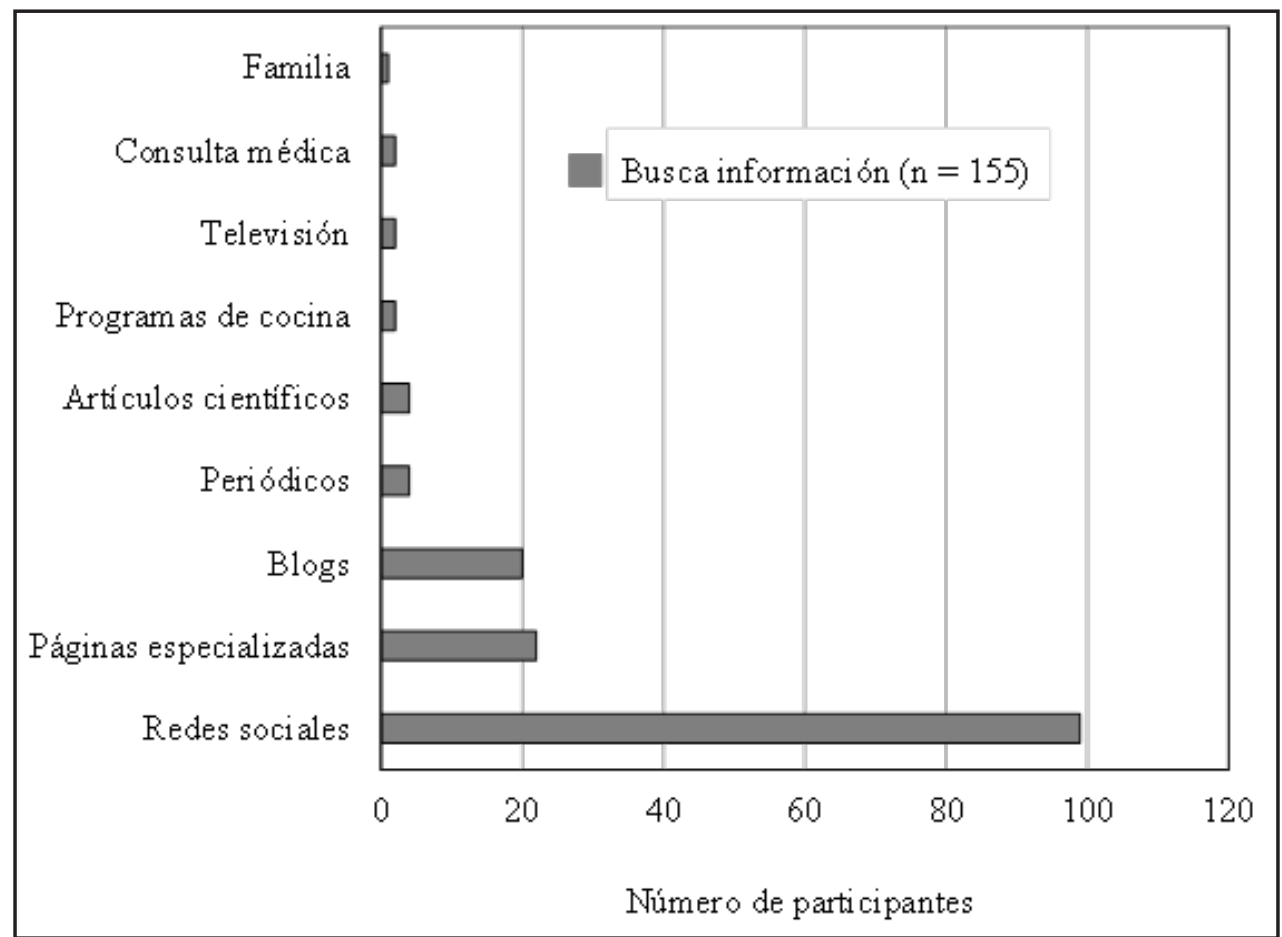

Figura 1. Medios utilizados por la población para buscar información relacionada con alimentación y nutrición.

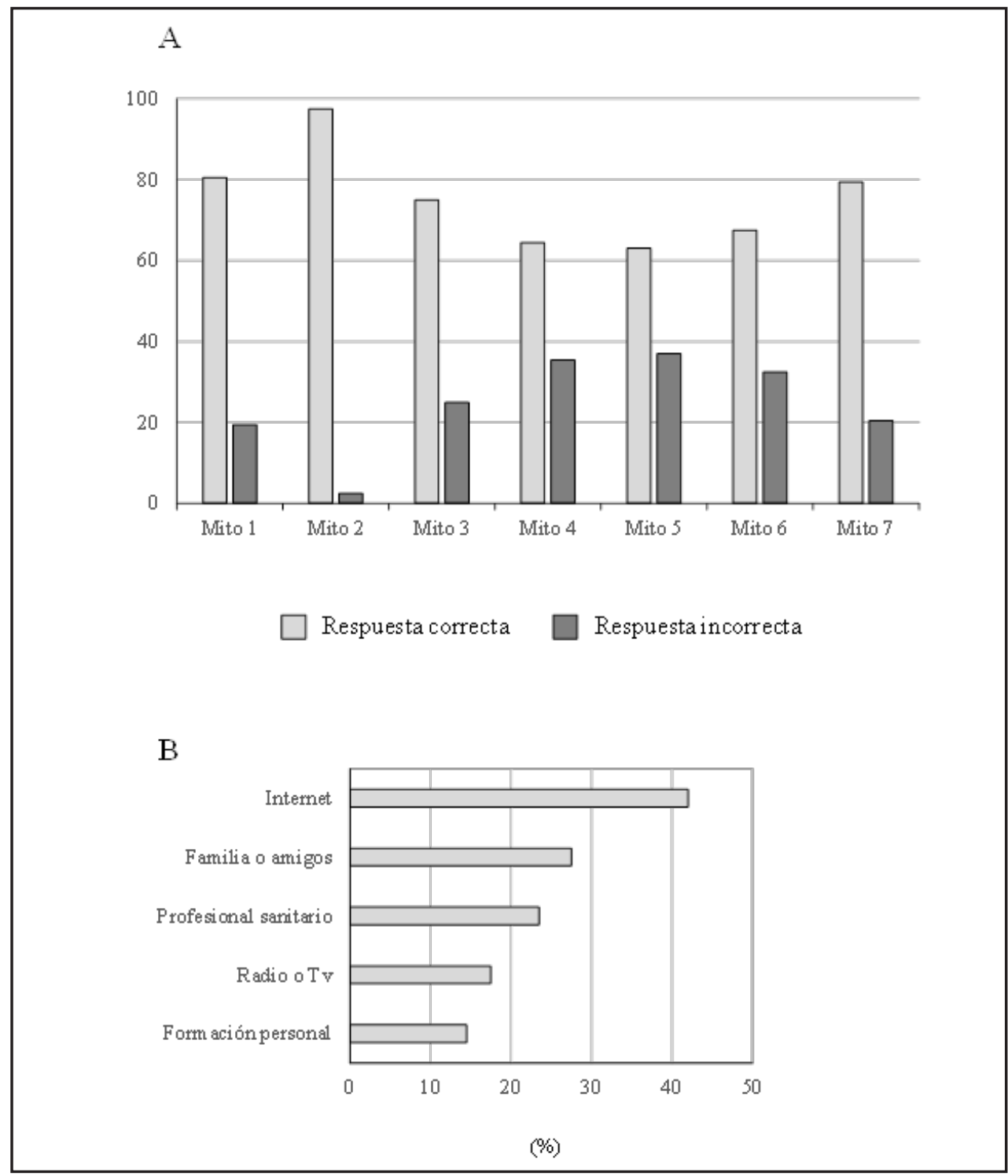

Figura 2. Porcentaje de aciertos a las preguntas relacionadas con los distintos mitos alimentarios (A) y medios indicados por los participantes para obtener dicha información (B) 
Mito 6. ¿El consumo de huevos aumenta el colesterol? "No, depende del resto de la dieta" era la respuesta esperada de este mito, seleccionada por el $67,5 \%$ de los sujetos (Figura 2A). En esta pregunta se demuestra que existen dudas en relación al binomio "huevos-colesterol", ya que un $27 \%$ respondió que este alimento aumenta el colesterol si se consumen más de tres huevos a la semana y un 5,5\% afirmó que lo aumenta sin importar el número de huevos consumidos.

Mito 7. ¿La leche sin lactosa es más saludable que la que sí la contiene? En esta pregunta, la respuesta "Sólo en el caso de las personas intolerantes a la lactosa" fue indicada por un $79,5 \%$ de los encuestados (Figura 2A). Del resto de los participantes, un $11,5 \%$ respondió que la leche sin lactosa no es más saludable y, por el contrario, un $9,5 \%$ afirmó que la leche sin lactosa es más saludable.

Según los encuestados, la obtención de esa información fue a través de internet $(42 \%)$, seguido de los consejos dados por familia y amigos $(27,5 \%)$, profesionales de la salud $(23,5 \%)$, adquiridos en radio o televisión $(17,5 \%)$ o por la propia formación personal $(14,5 \%)$ (Figura 2B). Si bien los hombres manifestaron una mayor búsqueda de información en internet $(32 \%$ más que las mujeres), las mujeres afirmaron recurrir en mayor medida a los consejos dados por la familia 0 amigos ( $30 \%$ más que los hombres).

\section{Discusión}

Teniendo en cuenta los mitos descritos por Sánchez (2016), podemos afirmar que una gran parte de las personas encuestadas conocía las respuestas correctas sobre los mitos, con porcentajes de acierto oscilando entre el $63,0 \%$ y el $97,5 \%$. Sin embargo, algunos sujetos aún no tienen claros estos conceptos.

Los mitos 1 y 2 fueron los que recibieron un mayor porcentaje de aciertos (80,5 y $97,5 \%$, respectivamente). De acuerdo con la literatura, no hay evidencias científicas que respalden que las dietas sin gluten sean más saludables, pudiendo incluso asociarse con efectos adversos (Niland \& Cash, 2018). Por el contrario, los efectos de la cantidad y calidad de la grasa alimentaria sobre la salud cardiovascular son ampliamente conocidos (Ros et al., 2015). Casi un 20\% de los participantes cree que la leche sin lactosa es más saludable (mito 7), mientras que un $25 \%$ optó por afirmar que los alimentos light son más saludables (mito 3). Sin embargo, respecto a este último mito, que contengan un número de calorías por unidad de peso inferior a los de su clase, no significa que no engorden, confusión que hace que sean consumidos sin límite ni control, olvidando que no son adelgazantes (Rapaille et al., 1995).
Los mitos 4-6 relacionados con el desayuno, las comidas necesarias diarias y el colesterol asociado al consumo de huevo, incluyen aspectos ampliamente evaluados por la comunidad científica (Spence, 2017; Zhuang et al., 2020). Sin embargo, son algo más desconocidos entre la población adulta y adolescente, ya que fueron los que causaron una mayor confusión entre los encuestados, con un porcentaje de respuestas incorrectas superior al 30\%. Cabe destacar un mayor porcentaje de aciertos en las mujeres, superando a los hombres en un $7-8 \%$ (mitos 2 y 4 ), $26 \%$ (mito 3 ) y 38 $41 \%$ (mitos 1 y 7 ). Los varones únicamente superaron las respuestas positivas en el mito 5 (10\% superior), siendo similares en ambos sexos para el mito 6 .

En línea con las afirmaciones de Gutiérrez-Sánchez (2016), el medio de comunicación más utilizado por parte del grupo poblacional encuestado para informarse sobre alimentación y nutrición fue internet. Principalmente debido a que este medio permite encontrar, de forma muy rápida y sencilla, toda la información de interés. En el año 2018, el $52 \%$ de la población de la Unión Europea (UE) utilizó internet para buscar información relacionada con la salud (Eurostat, 2018). En otros países como Australia también se ha indicado el uso creciente de internet como herramienta de búsqueda de aspectos relacionados con la nutrición, aumentándose en un 33,7\% en el año 2012 respecto a años anteriores (Pollard et al., 2015). Dentro de la plataforma de internet, las páginas más utilizadas son las redes sociales (Facebook, Instagram o Twitter), seguidas de páginas especializadas, blogs de nutrición y periódicos digitales (Figura 1). De forma similar, ha sido descrito que la mayoría de los ciudadanos que buscan información sobre salud, alimentación y nutrición utilizan buscadores de internet (85\%), webs especializadas $(20,8 \%)$, redes sociales $(11,4 \%)$ o blogs $(9,7 \%)$ (Pérez Rodrigo \& Gianzo Citores, 2018).

Las redes sociales son medios de comunicación donde las personas pueden compartir publicaciones de cualquier usuario. Por esta razón, pueden suponer un foco de divulgación errónea dependiendo de quién escriba la información. Carecen, además, de evaluaciones de calidad de la información publicada (Castillo Valenzuela et al., 2016). Es imprescindible que la población que utiliza las redes sociales para buscar información, tenga un filtro para identificar aquellas publicaciones que estén basadas en evidencias científicas. Es decir, cuáles son reales, y cuáles no. Un estudio sobre la veracidad de la información expuesta en las redes sociales reveló que la mayoría de los encuestados (95,2\%), sólo a veces cree en la información publicada en la red social (Castillo 
Valenzuela et al., 2016). Por el contrario, en las páginas especializadas, blogs de nutrición y periódicos digitales, los emisores deben ser profesionales de la salud o de la comunicación, transmitiendo datos y noticias veraces. Asegurar, por tanto, la veracidad de las noticias debería ser algo primordial para que la información recibida por los consumidores sea la correcta, especialmente si se trata de aspectos relevantes como la alimentación y la nutrición. Entender conceptos de nutrición puede ayudar a tomar decisiones apropiadas en la selección de alimentos y a corregir conceptos incorrectos o mitos sobre alimentación (Murga Eizagaechevarria, 2016).

Limitaciones del estudio. Este estudio tiene varias limitaciones que hay que tener en cuenta a la hora de interpretar los resultados. La muestra fue aleatoria, existiendo un criterio inicial para la selección de los primeros sujetos encuestados, pero no para el resto, sin incluir criterios de exclusión. Aunque los primeros participantes no tenían un especial conocimiento sobre la materia objeto de la encuesta, esta certeza no puede extrapolarse completamente al resto de los encuestados. Esto supone una limitación en relación a la representatividad de la muestra en la población adulta y adolescente general. Por otra parte, la mayor participación de mujeres con respecto a los hombres hace que el grupo encuestado no sea del todo homogéneo y representativo. La encuesta no tenía como objetivos evaluar el nivel educativo de los participantes, de nuevo no pudiendo descartarse el conocimiento sobre el tema en cuestión. Finalmente, dado que es un estudio observacional transversal, no se puede establecer ni descartar una relación de causalidad.

\section{Conclusión}

El $78 \%$ de la población adulta y adolescente encuestada afirma buscar información sobre aspectos relacionados con la alimentación, recurriendo principalmente a internet para realizar dicha búsqueda. Acceder a noticias con falta de veracidad podría ser la causa de la difusión de ciertos mitos alimentarios. A pesar de que los sujetos encuestados en el presente estudio no han mostrado una gran confusión en relación a determinados mitos sobre los alimentos, es importante concienciar a la sociedad de que hay que contrastar la información recibida y tener espíritu crítico. De esta manera, se podrían erradicar mitos y noticias falsas sobre aspectos relacionados con la alimentación y la nutrición.

Contribuciones de los autores

Los autores participaron igualmente en la elaboración del manuscrito y aprobaron la versión final presentada.

\section{Financiación}

Esta investigación no recibió financiación.

Declaración de disponibilidad de datos

Los datos presentados en este estudio pueden ser solicitados al autor de correspondencia.

Conflicto de interés

Los autores declaran que no hay conflicto de interés.

\section{Referencias bibliográficas}

Alzate Narváez, T. (2013). Consejos dietéticos y nutricionales en la prensa española. Revista Española de Comunicación en Salud, 4(1), 1726.

Barreiro, B. (2019). Buceando en las tendencias alimentarias de los españoles. Alimentos de España. Retrieved from https://www.mapa.gob.es/es/ prensa/ultimas-noticias/el-ministerio-de-agriculturapesca-y-alimentaci\%C3\%B3n-presenta-un-estudiosobre-el-comportamiento-sociol\%C3\%B3gico-de-losespa\%C3\%B1oles-y-su-vinculaci\%C3\%B3n-con-/ tcm:30-507218

Berg, S. (1988). Snowball sampling. In S. Kotz \& N. L. Johnson (Eds.), Encyclopedia of Statistical Sciences (pp. 528-532). New Hersey, USA: WileyInterscience.

Carretero Sánchez, M. (2016). La comunicación de la nutrición en España. Tesis de doctorado inédita. Madrid, España: Universidad Complutense de Madrid.

Castillo Sánchez, M. D., León Espinosa de los Monteros, M.T., \& Naranjo Rodríguez, J. A. (2001). Creencias erróneas sobre alimentación. Medicina General, 33, 346-350.

Castillo Valenzuela, M., Cruces Hernández, L., \& Guerra Goglios, M. (2016). La veracidad de la información expuesta en las redes sociales. Revista Educación y Tecnología, 9, 26-40.

Echenove, M., \& Puértolas, S. (2019). Healthy Food \& Brands. Retrieved from: https://www. programapublicidad.com/234695-2/

Eurostat. (2018). What do you use the internet for? Retrieved from: https://ec.europa.eu/eurostat/web/ products-eurostat-news/-/DDN-20190124-1

Fundación Española para la Ciencia y la Tecnología. (2018). Encuesta de percepción Social de la Ciencia. Informe de resultados. Madrid, España: 
Ministerio de Ciencia, Innovación y Universidades.

Feldman, S., \& Marks, V. (2016). Panic Nation: Exposing the myths we're told about food and health. England: John Blake Publishing.

García-García, J. A., Reding-Bernal, A., \& LópezAlvarenga, J. C. (2013). Cálculo del tamaño de la muestra en investigación en educación médica. Investigación en Educación Médica, 2(8), 217224. doi: 10.1016/S2007-5057(13)72715-7.

Gutiérrez-Sánchez, I. (2016). La comunicación en alimentación y salud: una responsabilidad social. Revista Española de Comunicación en Salud, S1, S11-S14. doi: 10.20318/recs.2016.3117

Ipsos (2018). Los españoles, los europeos que más caen en las trampas de las "Fake News". Retreived from: https://www.ipsos.com/es-es/losespanoles-los-europeos-que-mas-caen-en-lastrampas-de-las-fake-news

Murga Eizagaechevarria, N. (2016). e-ciudadanos, e-salud y redes sociales Organizarse y formarse en alimentación y salud. Revista Española de Comunicación en Salud, S1, S139-S144. doi: 10.20318/recs.2016.3133

Niland, B., \& Cash, B. D. (2018). Health Benefits and Adverse Effects of a Gluten-Free Diet in Non-Celiac Disease Patients. Gastroenterology \& Hepatology, 14(2), 82-91.

Pérez Rodrigo, C., \& Gianzo Citores, M. (2018). Alimentación 3.0: aspectos de interés en la práctica diaria. Nutrición Hospitalaria, 35, 85-95. doi: 10.20960/nh.2132

Pollard, C. M., Pulker, C. E., Meng, X., Kerr, D. A., \& Scott, J. A. (2015). Who Uses the Internet as a Source of Nutrition and Dietary Information? An Australian Population Perspective. Journal of Medicine Internet Research, 17, e209. doi: 10.2196/jmir.4548

Rapaille, A., Gonze, M., \& Van der Schueren, F. (1995). Formulating sugar-free chocolate products with maltitol. Food Technology, 49(7), 51-54.

Ros, E., López-Miranda, J., Picó, C., Rubio, M. A., Babio, N., Sala-Vila, A., Pérez-Jiménez, D., Escrich, E., Bulló, M., Solanas, M., Gil Hernández, A., \& SalasSalvadó, J. (2015). Consenso sobre las grasas y aceites en la alimentación de la población española adulta; postura de la Federación Española de Sociedades de Alimentación, Nutrición y Dietética (FESNAD). Nutrición Hospitalaria, 32(2), 435-477. doi: 10.3305/nh.2015.32.2.9202.

Sánchez, A. (2016). Mi dieta cojea. Los mitos sobre nutrición que te han hecho creer. Madrid, España: Editorial Paidós.

Spence, C. (2017). Breakfast: The most important meal of the day? International Journal of Gastronomy and Food Science, 8, 1-6.

Zhuang, P., Jiao, J., Wu, F., Mao, L., \& Zhang, Y. (2020). Egg and egg-sourced cholesterol consumption in relation to mortality: Findings from population-based nationwide cohort. Clinical Nutrition, 39(11), 35203527. doi: 10.1016/j.clnu.2020.03.019. 